\title{
Research Facilities Construction Grants
}

National Cancer Institute

\section{Source}

National Cancer Institute. Research Facilities Construction Grants. NCI Thesaurus. Code C20018.

Support to expand, remodel, renovate, or alter biomedical or behavioral research facilities. 